\title{
On Multiset Ordering
}

\author{
Grzegorz Bancerek \\ Association of Mizar Users \\ Białystok, Poland
}

\begin{abstract}
Summary. Formalization of a part of 11. Unfortunately, not all is possible to be formalized. Namely, in the paper there is a mistake in the proof of Lemma 3. It states that there exists $x \in M_{1}$ such that $M_{1}(x)>N_{1}(x)$ and $\left(\forall y \in N_{1}\right) x \nprec y$. It should be $M_{1}(x) \geqslant N_{1}(x)$. Nevertheless we do not know whether $x \in N_{1}$ or not and cannot prove the contradiction. In the article we referred to [8], 9] and 10.
\end{abstract}

MSC: 06F05 03B35

Keywords: ordering; Dershowitz-Manna ordering

MML identifier: BAGORD_2, version: 8.1.04 5.36.1267

\section{Preliminaries}

Now we state the propositions:

(1) Let us consider natural numbers $m, n$. Then $n=m-^{\prime}\left(m-{ }^{\prime} n\right)+\left(n-{ }^{\prime} m\right)$.

(2) Let us consider natural numbers $n, m$. Then $m-{ }^{\prime} n \geqslant m-n$.

Let us consider natural numbers $m, n, x, y$. Now we state the propositions:

(3) If $n=m-{ }^{\prime} x+y$, then $m-{ }^{\prime} n \leqslant x$ and $n-{ }^{\prime} m \leqslant y$. The theorem is a consequence of $(2)$.

(4) If $x \leqslant m$ and $n=m-^{\prime} x+y$, then $x-^{\prime}\left(m-{ }^{\prime} n\right)=y-^{\prime}\left(n-{ }^{\prime} m\right)$. The theorem is a consequence of (3).

Now we state the propositions:

(5) Let us consider natural numbers $k, x_{1}, x_{2}, y_{1}, y_{2}$. Suppose $x_{2} \leqslant k$ and $x_{1} \leqslant k-{ }^{\prime} x_{2}+y_{2}$. Then

(i) $x_{2}+\left(x_{1}-{ }^{\prime} y_{2}\right) \leqslant k$, and 
(ii) $k-^{\prime} x_{2}+y_{2}-^{\prime} x_{1}+y_{1}=k-^{\prime}\left(x_{2}+\left(x_{1}-^{\prime} y_{2}\right)\right)+\left(y_{2}-^{\prime} x_{1}+y_{1}\right)$.

Proof: $x_{2}+\left(x_{1}-{ }^{\prime} y_{2}\right) \leqslant k$ by [12, (8)].

(6) Let us consider natural numbers $x$, $y$. If $x+y>0$, then $x>0$ or $y>0$.

From now on $a, b$ denote objects and $I, J$ denote sets.

Let us consider $I$. Let $J$ be a non empty set. Let us note that every function from $I$ into $J$ is total and there exists a relational structure which is asymmetric, transitive, and non empty.

Let us consider $I$. One can verify that there exists a binary relation on $I$ which is asymmetric and transitive.

Let $R$ be a transitive relational structure. Observe that the internal relation of $R$ is transitive.

Let $R$ be an asymmetric relational structure. Let us observe that the internal relation of $R$ is asymmetric.

Let us consider $I$. Let $p, q$ be $I$-valued finite sequences. Let us observe that $p^{\frown} q$ is $I$-valued.

Now we state the proposition:

(7) Let us consider finite sequences $p, q$. Suppose $p^{\wedge} q$ is $I$-valued. Then

(i) $p$ is $I$-valued, and

(ii) $q$ is $I$-valued.

Let us consider $I$. Let $f$ be an $I$-valued finite sequence and $n$ be a natural number. Let us note that $f\lceil n$ is $I$-valued.

Now we state the propositions:

(8) Let us consider a finite sequence $p$. Suppose $a \in \operatorname{rng} p$. Then there exist

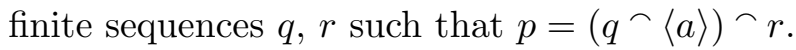

(9) Let us consider finite sequences $p, q$. Then $p \subset q$ if and only if len $p<\operatorname{len} q$ and for every natural number $i$ such that $i \in \operatorname{dom} p$ holds $p(i)=q(i)$.

(10) Let us consider finite sequences $p, q, r$. Then $r^{\frown} p \subset r^{\frown} q$ if and only if $p \subset q$.

Proof: If $r^{\frown} p \subset r^{\frown} q$, then $p \subset q$ by [4, (22)], (9), [15, (30)], [4, (28)].

Let $R$ be an asymmetric, non empty relational structure and $x, y$ be elements of $R$. Let us observe that the predicate $x \leqslant y$ is asymmetric.

Now we state the proposition:

(11) Let us consider an asymmetric, non empty relational structure $R$, and elements $x, y$ of $R$. Then $x \leqslant y$ if and only if $x<y$. 


\section{Relational Extension}

Let us consider $I$.

A multiset of $I$ is an element of $I^{\otimes}$. Observe that every multiset of $I$ is $I$-defined and natural-valued and every multiset of $I$ is total.

Let $m$ be a natural-valued function. Let us note that the functor support $m$ is defined by the term

(Def. 1) $m^{-1}(\mathbb{N} \backslash\{0\})$.

Let us consider $I$. One can check that every multiset of $I$ is finite-support.

Now we state the propositions:

(12) $a$ is a multiset of $I$ if and only if $a$ is a bag of $I$.

(13) $1_{I \otimes}=$ EmptyBag $I$.

Let $R$ be a relational structure and $x, y$ be elements of $R$. We say that $x \equiv y$ if and only if

(Def. 2) $x \nless y$ and $y \nless x$.

Observe that the predicate is symmetric.

We consider relational multiplicative magmas which extend multiplicative magmas and relational structures and are systems

〈a carrier, a multiplication, an internal relation〉

where the carrier is a set, the multiplication is a binary operation on the carrier, the internal relation is a binary relation on the carrier.

We consider relational monoids which extend multiplicative loop structures and relational structures and are systems

$$
\text { 〈a carrier, a multiplication, a one, an internal relation〉 }
$$

where the carrier is a set, the multiplication is a binary operation on the carrier, the one is an element of the carrier, the internal relation is a binary relation on the carrier.

Let $M$ be a multiplicative loop structure.

A relational extension of $M$ is a relational monoid and is defined by

(Def. 3) the multiplicative loop structure of $i t=$ the multiplicative loop structure of $M$.

Let $M$ be a non empty multiplicative loop structure. Let us observe that every relational extension of $M$ is non empty.

Let $M$ be a multiplicative loop structure. One can check that there exists a relational extension of $M$ which is strict.

Let us consider a multiplicative loop structure $N$ and a relational extension $M$ of $N$. Now we state the propositions: 
(14) $a$ is an element of $M$ if and only if $a$ is an element of $N$.

(15) $1_{N}=1_{M}$.

Let us consider $I$. Let $M$ be a relational extension of $I^{\otimes}$. Let us observe that every element of $M$ is function-like and relation-like and every element of $M$ is $I$-defined, natural-valued, and finite-support and every element of $M$ is total.

Now we state the proposition:

(16) Let us consider a relational extension $M$ of $I^{\otimes}$. Then the carrier of $M=$ Bags $I$. The theorem is a consequence of (12) and (14).

The scheme RelEx deals with a non empty multiplicative loop structure $\mathcal{M}$ and a binary predicate $\mathcal{R}$ and states that

(Sch. 1) There exists a strict relational extension $N$ of $\mathcal{M}$ such that for every elements $x, y$ of $N, x \leqslant y$ iff $\mathcal{R}[x, y]$.

Now we state the proposition:

(17) Let us consider a multiplicative loop structure $N$, and strict relational extensions $M_{1}, M_{2}$ of $N$. Suppose for every elements $m, n$ of $M_{1}$ for every elements $x, y$ of $M_{2}$ such that $m=x$ and $n=y$ holds $m \leqslant n$ iff $x \leqslant y$. Then $M_{1}=M_{2}$.

PRoOF: The internal relation of $M_{1}=$ the internal relation of $M_{2}$ by [7, $(87)]$.

\section{Dershowitz-Manna Order}

Let $R$ be a non empty relational structure. The Dershowitz-Manna order $R$ yielding a strict relational extension of (the carrier of $R)^{\otimes}$ is defined by

(Def. 4) for every elements $m, n$ of it, $m \leqslant n$ iff there exist elements $x, y$ of it such that $1_{i t} \neq x \mid n$ and $m=n-{ }^{\prime} x+y$ and for every element $b$ of $R$ such that $y(b)>0$ there exists an element $a$ of $R$ such that $x(a)>0$ and $b \leqslant a$.

Now we state the proposition:

(18) Let us consider bags $m, n$ of $I$. Then $n=m-^{\prime}\left(m-{ }^{\prime} n\right)+\left(n-{ }^{\prime} m\right)$. The theorem is a consequence of (1).

Let us consider bags $m, n, x, y$ of $I$. Now we state the propositions:

(19) If $n=m-^{\prime} x+y$, then $m-^{\prime} n \mid x$ and $n-^{\prime} m \mid y$. The theorem is a consequence of $(3)$.

(20) If $x \mid m$ and $n=m-^{\prime} x+y$, then $x-^{\prime}\left(m-{ }^{\prime} n\right)=y-^{\prime}\left(n-{ }^{\prime} m\right)$. The theorem is a consequence of (4).

Now we state the propositions: 
(21) Let us consider bags $m, x, y$ of $I$. If $x \mid m$ and $x \neq y$, then $m \neq m-^{\prime} x+y$.

(22) Let us consider a non empty set $I$, a binary relation $R$ on $I$, and a reduction sequence $r$ w.r.t. $R$. If len $r>1$, then $r($ len $r) \in I$.

(23) Let us consider an asymmetric, transitive binary relation $R$ on $I$. Then every reduction sequence w.r.t. $R$ is one-to-one.

Proof: For every natural numbers $i, j$ such that $i>j$ and $i, j \in \operatorname{dom} r$ holds $r(i) \neq r(j)$ by [1, (13)], [13, (22)], [1, (11)], [15, (25)].

(24) Let us consider an asymmetric, transitive, non empty relational structure $R$, and a set $X$. Suppose $X$ is finite and there exists an element $x$ of $R$ such that $x \in X$. Then there exists an element $x$ of $R$ such that $x$ is maximal in $X$.

Proof: Reconsider $X_{1}=X$ as a finite set. Set $Y=\{r$, where $r$ is an element of $X_{1}{ }^{*}: r$ is a reduction sequence w.r.t. the internal relation of $R$ \}. Define $\mathcal{P}$ [natural number] $\equiv$ there exists a reduction sequence $r$ w.r.t. the internal relation of $R$ such that $r \in Y$ and len $r=\$_{1}$. For every natural number $k$ such that $\mathcal{P}[k]$ holds $k \leqslant \overline{\overline{X_{1}}}$ by (23), [1, (43)]. $\mathcal{P}[1]$ by [2, (6)], [4, (74), (39)]. Consider $k$ being a natural number such that $\mathcal{P}[k]$ and for every natural number $n$ such that $\mathcal{P}[n]$ holds $n \leqslant k$ from [1, Sch. 6]. Consider $r$ being a reduction sequence w.r.t. the internal relation of $R$ such that $r \in Y$ and len $r=k$. Consider $q$ being an element of $X_{1}{ }^{*}$ such that $r=q$ and $q$ is a reduction sequence w.r.t. the internal relation of $R$.

(25) Let us consider bags $m, n$ of $I$. Then $m-{ }^{\prime} n \mid m$.

Let us consider $I$. Note that every element of Bags $I$ is function-like and relation-like.

Now we state the proposition:

(26) Let us consider bags $m, n$ of $I$. Then

(i) $m-{ }^{\prime} n \neq \operatorname{EmptyBag} I$, or

(ii) $m=n$, or

(iii) $n-{ }^{\prime} m \neq$ EmptyBag $I$.

Let $R$ be an asymmetric, transitive, non empty relational structure. Let us observe that the Dershowitz-Manna order $R$ is defined by

(Def. 5) for every elements $m, n$ of it, $m \leqslant n$ iff $m \neq n$ and for every element $a$ of $R$ such that $m(a)>n(a)$ there exists an element $b$ of $R$ such that $a \leqslant b$ and $m(b)<n(b)$.

Now we state the proposition:

(27) Let us consider bags $k, x_{1}, x_{2}, y_{1}, y_{2}$ of $I$. Suppose $x_{2} \mid k$ and $x_{1} \mid$ $k-{ }^{\prime} x_{2}+y_{2}$. Then 
(i) $x_{2}+\left(x_{1}-{ }^{\prime} y_{2}\right) \mid k$, and

(ii) $k-{ }^{\prime} x_{2}+y_{2}-^{\prime} x_{1}+y_{1}=k-{ }^{\prime}\left(x_{2}+\left(x_{1}-^{\prime} y_{2}\right)\right)+\left(y_{2}-^{\prime} x_{1}+y_{1}\right)$.

The theorem is a consequence of (5).

Let $R$ be an asymmetric, transitive, non empty relational structure. Let us observe that the Dershowitz-Manna order $R$ is asymmetric and transitive.

Let us consider $I$. The functor DivOrder $(I)$ yielding a binary relation on Bags $I$ is defined by

(Def. 6) for every bags $b_{1}, b_{2}$ of $I,\left\langle b_{1}, b_{2}\right\rangle \in$ it iff $b_{1} \neq b_{2}$ and $b_{1} \mid b_{2}$.

Now we state the proposition:

(28) Let us consider bags $a, b, c$ of $I$. If $a|b| c$, then $a \mid c$.

Let us consider $I$. Note that $\operatorname{DivOrder}(I)$ is asymmetric and transitive.

Let us consider an asymmetric, transitive, non empty relational structure $R$. Now we state the propositions:

(29) DivOrder(the carrier of $R) \subseteq$ the internal relation of the DershowitzManna order $R$. The theorem is a consequence of (12) and (14).

(30) Suppose the internal relation of $R$ is empty. Then the internal relation of the Dershowitz-Manna order $R=\operatorname{DivOrder}($ the carrier of $R$ ). The theorem is a consequence of (29).

Now we state the proposition:

(31) Let us consider asymmetric, transitive, non empty relational structures $R_{1}, R_{2}$. Suppose the carrier of $R_{1}=$ the carrier of $R_{2}$ and the internal relation of $R_{1} \subseteq$ the internal relation of $R_{2}$. Then the internal relation of the Dershowitz-Manna order $R_{1} \subseteq$ the internal relation of the DershowitzManna order $R_{2}$. The theorem is a consequence of (12) and (14).

\section{Monoidal Order}

Let us consider $I$. Let $f$ be a (Bags $I$ )-valued finite sequence. The functor $\sum f$ yielding a bag of $I$ is defined by

(Def. 7) there exists a function $F$ from $\mathbb{N}$ into Bags $I$ such that $i t=F(\operatorname{len} f)$ and $F(0)=$ EmptyBag $I$ and for every natural number $i$ and for every bag $b$ of $I$ such that $i<\operatorname{len} f$ and $b=f(i+1)$ holds $F(i+1)=F(i)+b$.

Now we state the proposition:

(32) $\sum \varepsilon_{\text {Bags } I}=\operatorname{EmptyBag} I$.

Let us consider $I$. Let $b$ be a bag of $I$. One can verify that $\langle b\rangle$ is (Bags $I$ )valued as a finite sequence.

Now we state the proposition: 
(33) Let us consider a (Bags $I)$-valued finite sequence $p$, and a bag $b$ of $I$.

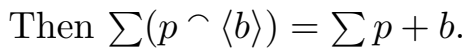

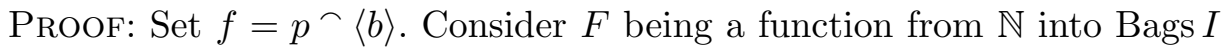
such that $\sum f=F(\operatorname{len} f)$ and $F(0)=$ EmptyBag $I$ and for every natural number $i$ and for every bag $b$ of $I$ such that $i<\operatorname{len} f$ and $b=f(i+1)$ holds $F(i+1)=F(i)+b$. Consider $F_{1}$ being a function from $\mathbb{N}$ into Bags $I$ such that $\sum p=F_{1}(\operatorname{len} p)$ and $F_{1}(0)=$ EmptyBag $I$ and for every natural number $i$ and for every bag $b$ of $I$ such that $i<\operatorname{len} p$ and $b=p(i+1)$ holds $F_{1}(i+1)=F_{1}(i)+b$. Define $\mathcal{P}$ [natural number] $\equiv$ if $\$_{1} \leqslant$ len $p$, then $F\left(\$_{1}\right)=F_{1}\left(\$_{1}\right)$. For every natural number $i$ such that $\mathcal{P}[i]$ holds $\mathcal{P}[i+1]$ by [5, (16)], [1, (13), (11)], [15, (25)]. For every natural number $i, \mathcal{P}[i]$ from [1, Sch. 2].

From now on $b$ denotes a bag of $I$.

Now we state the propositions:

(34) $\sum\langle b\rangle=b$. The theorem is a consequence of (33) and (32).

(35) Let us consider (Bags $I$ )-valued finite sequences $p, q$. Then $\sum\left(p^{\frown} q\right)=$ $\sum p+\sum q$.

Proof: Set $f=p^{\frown} q$. Consider $F$ being a function from $\mathbb{N}$ into Bags $I$ such that $\sum f=F(\operatorname{len} f)$ and $F(0)=$ EmptyBag $I$ and for every natural number $i$ and for every bag $b$ of $I$ such that $i<\operatorname{len} f$ and $b=f(i+1)$ holds $F(i+1)=F(i)+b$. Consider $F_{1}$ being a function from $\mathbb{N}$ into Bags $I$ such that $\sum p=F_{1}(\operatorname{len} p)$ and $F_{1}(0)=$ EmptyBag $I$ and for every natural number $i$ and for every bag $b$ of $I$ such that $i<\operatorname{len} p$ and $b=p(i+1)$ holds $F_{1}(i+1)=F_{1}(i)+b$. Consider $F_{2}$ being a function from $\mathbb{N}$ into Bags $I$ such that $\sum q=F_{2}(\operatorname{len} q)$ and $F_{2}(0)=$ EmptyBag $I$ and for every natural number $i$ and for every bag $b$ of $I$ such that $i<\operatorname{len} q$ and $b=q(i+1)$ holds $F_{2}(i+1)=F_{2}(i)+b$. Define $\mathcal{P}$ [natural number] $\equiv$ if $\$_{1} \leqslant \operatorname{len} p$, then $F\left(\$_{1}\right)=F_{1}\left(\$_{1}\right)$. For every natural number $i$ such that $\mathcal{P}[i]$ holds $\mathcal{P}[i+1]$ by [4, (22)], [1, (11), (13)], [15, (25)]. For every natural number $i, \mathcal{P}[i]$ from [1, Sch. 2]. Define $\mathcal{Q}\left[\right.$ natural number] $\equiv$ if $\$_{1} \leqslant \operatorname{len} q$, then $F\left(\operatorname{len} p+\$_{1}\right)=\sum p+F_{2}\left(\$_{1}\right)$. For every natural number $i$ such that $\mathcal{Q}[i]$ holds $\mathcal{Q}[i+1]$ by [4, (22)], [1, (13), (11)], [15, (25)]. For every natural number $i, \mathcal{Q}[i]$ from [1, Sch. 2].

Let us consider a (Bags $I$ )-valued finite sequence $p$. Now we state the propositions:

(36) $\sum(\langle b\rangle \frown p)=b+\sum p$. The theorem is a consequence of (35) and (34).

(37) If $b \in \operatorname{rng} p$, then $b \mid \sum p$. The theorem is a consequence of $(8),(7),(33)$, and (35).

Now we state the proposition: 
(38) Let us consider a (Bags $I$ )-valued finite sequence $p$, and an object $i$. Suppose $i \in \operatorname{support} \sum p$. Then there exists $b$ such that

(i) $b \in \operatorname{rng} p$, and

(ii) $i \in \operatorname{support} b$.

Proof: Define $\mathcal{P}$ [natural number] $\equiv$ for every (Bags $I$ )-valued finite sequence $p$ such that len $p=\$_{1}$ for every object $i$ such that $i \in \operatorname{support} \sum p$ there exists $b$ such that $b \in \operatorname{rng} p$ and $i \in \operatorname{support} b . \mathcal{P}[0]$. For every natural number $j$ such that $\mathcal{P}[j]$ holds $\mathcal{P}[j+1]$ by [3, (3)], (7), [4, (40)], [15, (25)]. For every natural number $j, \mathcal{P}[j]$ from [1, Sch. 2].

Let us consider $I$ and $b$.

A partition of $b$ is a (Bags $I$ )-valued finite sequence and is defined by (Def. 8) $b=\sum i t$.

Observe that the functor $\langle b\rangle$ yields a partition of $b$. Let $R$ be a relational structure, $M$ be a relational extension of (the carrier of $R)^{\otimes}, b$ be an element of $M$, and $p$ be a partition of $b$. We say that $p$ is co-ordered if and only if

(Def. 9) for every natural number $i$ such that $i, i+1 \in \operatorname{dom} p$ for every elements $b_{1}, b_{2}$ of $M$ such that $b_{1}=p(i)$ and $b_{2}=p(i+1)$ holds $b_{2} \leqslant b_{1}$.

Let $R$ be a non empty relational structure and $b$ be a bag of the carrier of $R$. We say that $p$ is ordered if and only if

(Def. 10) for every bag $m$ of the carrier of $R$ such that $m \in \operatorname{rng} p$ for every element $x$ of $R$ such that $m(x)>0$ holds $m(x)=b(x)$ and for every bag $m$ of the carrier of $R$ such that $m \in \operatorname{rng} p$ for every elements $x, y$ of $R$ such that $m(x)>0$ and $m(y)>0$ and $x \neq y$ holds $x \equiv y$ and for every bag $m$ of the carrier of $R$ such that $m \in \operatorname{rng} p$ holds $m \neq$ EmptyBag(the carrier of $R$ ) and for every natural number $i$ such that $i, i+1 \in \operatorname{dom} p$ for every element $x$ of $R$ such that $p_{i+1}(x)>0$ there exists an element $y$ of $R$ such that $p_{i}(y)>0$ and $x \leqslant y$.

In the sequel $R$ denotes an asymmetric, transitive, non empty relational structure, $a, b, c$ denote bags of the carrier of $R$, and $x, y, z$ denote elements of $R$.

Now we state the propositions:

(39) $\langle a\rangle$ is ordered if and only if $a \neq$ EmptyBag(the carrier of $R$ ) and for every $x$ and $y$ such that $a(x)>0$ and $a(y)>0$ and $x \neq y$ holds $x \equiv y$.

(40) Let us consider a (Bags $I$ )-valued finite sequence $p$, and bags $a, b$ of $I$. Then $\langle a\rangle^{\frown} p$ is a partition of $b$ if and only if $a \mid b$ and $p$ is a partition of $b-{ }^{\prime} a$. The theorem is a consequence of (36).

From now on $p$ denotes a partition of $b-^{\prime} a$ and $q$ denotes a partition of $b$. Now we state the proposition: 
(41) If $q=\langle a\rangle^{\frown} p$ and $q$ is ordered, then $p$ is ordered. The theorem is a consequence of (37) and (25).

Let us consider $I$. Let $m$ be a bag of $I$ and $J$ be a set. The functor $m\lceil J$ yielding a bag of $I$ is defined by

(Def. 11) for every object $i$ such that $i \in I$ holds if $i \in J$, then $i t(i)=m(i)$ and if $i \notin J$, then $i t(i)=0$.

From now on $J$ denotes a set and $m$ denotes a bag of $I$.

Now we state the propositions:

(42) $\operatorname{support}(m\lceil J)=J \cap \operatorname{support} m$.

(43) $m\lceil J+m \uparrow(I \backslash J)=m$.

(44) $m\lceil J \mid m$.

(45) If support $m \subseteq J$, then $m\lceil J=m$.

(46) $\operatorname{support}\left(m-{ }^{\prime} m\lceil J)=\operatorname{support} m \backslash J\right.$.

(47) If $q$ is ordered and $q=\langle a\rangle^{\frown} p$ and $a(x)>0$, then $a(x)=b(x)$.

(48) If $q$ is ordered and $q=\langle a\rangle^{\wedge} p$ and $a(x)>0$ and $a(y)>0$ and $x \neq y$, then $x \equiv y$.

(49) If $q$ is ordered and $q=\langle a\rangle^{\frown} p$, then $a \neq \operatorname{EmptyBag}$ (the carrier of $R$ ).

(50) Let us consider a bag $c$ of the carrier of $R$, and a (Bags(the carrier of $R)$ )-valued finite sequence $r$. Suppose $q$ is ordered and $q=\langle a, c\rangle^{\frown} r$ and $c(y)>0$. Then there exists $x$ such that

(i) $a(x)>0$, and

(ii) $y \leqslant x$.

(51) If $x \in I$ and for every $y$ such that $y \in I$ and $y \neq x$ holds $x \equiv y$, then $x$ is maximal in $I$.

(52) If $q$ is ordered and $q=\langle a\rangle^{\frown} p$ and $c \in \operatorname{rng} p$ and $c(x)>0$, then there exists $y$ such that $a(y)>0$ and $x \leqslant y$.

Proof: Consider $i$ being an object such that $i \in \operatorname{dom} p$ and $c=p(i)$. Define $\mathcal{P}$ [natural number] $\equiv$ if $\$_{1} \in \operatorname{dom} p$, then for every $x$ such that $p_{\$_{1}}(x)>0$ there exists $y$ such that $a(y)>0$ and $x \leqslant y . \mathcal{P}[1]$ by [4, (28)], [15. (25)], 44, (40)]. For every natural number $i$ such that $i \geqslant 1$ and $\mathcal{P}[i]$ holds $\mathcal{P}[i+1]$ by [1, (13)], [15, (25)], [4, (28)], [16, (3)]. For every natural number $i$ such that $i \geqslant 1$ holds $\mathcal{P}[i]$ from [1, Sch. 8].

Let us assume that $q$ is ordered and $q=\langle a\rangle^{\frown} p$. Now we state the propositions:

(53) $x$ is maximal in support $b$ if and only if $a(x)>0$.

Proof: $a \mid \sum q=b$. There exists no $y$ such that $y \in \operatorname{support} b$ and $x<y$ by (48), (38), [4, (31), (39)]. 
(54) $a=b\lceil\{x: x$ is maximal in support $b\}$. The theorem is a consequence of (53) and (47).

Now we state the propositions:

(55) Let us consider a (Bags $I$ )-valued finite sequence $p$. Suppose $\sum p=$ EmptyBag $I$ and for every bag $a$ of $I$ such that $a \in \operatorname{rng} p$ holds $a \neq$ EmptyBag $I$. Then $p=\emptyset$. The theorem is a consequence of (37).

(56) Let us consider bags $a, b$ of $I$. If $a \neq$ EmptyBag $I$, then $a+b \neq$ EmptyBag $I$.

(57) Let us consider partitions $p, q$ of $b$. If $p$ is ordered and $q$ is ordered, then $p=q$.

Proof: Define $\mathcal{P}$ [natural number] $\equiv$ for every $b$ and $q$ such that len $q=\$_{1}$ and $q$ is ordered for every partition $p$ of $b$ such that $p$ is ordered holds $q=p$. $\mathcal{P}[0]$. For every natural number $i$ such that $\mathcal{P}[i]$ holds $\mathcal{P}[i+1]$ by [5, (130)], (40), (49), (36). For every natural number $i, \mathcal{P}[i]$ from [1, Sch. 2].

Let us consider $I$. Let $a, b$ be bags of $I$. One can verify that the functor $\langle a$, $b\rangle$ yields an element of Bags $I \times \operatorname{Bags} I$. Now we state the proposition:

(58) Suppose $a \neq$ EmptyBag(the carrier of $R$ ). Then $\{x: x$ is maximal in support $a\} \neq \emptyset$. The theorem is a consequence of (24).

Let us consider $R$ and $b$. The ordered partition of $b$ yielding a (Bags(the carrier of $R$ ))-valued finite sequence is defined by

(Def. 12) there exist functions $F, G$ from $\mathbb{N}$ into Bags(the carrier of $R$ ) such that $F(0)=b$ and $G(0)=$ EmptyBag(the carrier of $R$ ) and for every natural number $i, G(i+1)=F(i)\{\{x: x$ is maximal in $\operatorname{support}(F(i))\}$ and $F(i+$ $1)=F(i)-{ }^{\prime} G(i+1)$ and there exists a natural number $i$ such that $F(i)=\operatorname{EmptyBag}($ the carrier of $R)$ and $i t=G\lceil\operatorname{Seg} i$ and for every natural number $j$ such that $j<i$ holds $F(j) \neq \operatorname{EmptyBag}$ (the carrier of $R$ ).

One can verify that the ordered partition of $b$ yields a partition of $b$. Let us note that the ordered partition of $b$ is ordered as a partition of $b$.

Now we state the proposition:

(59) $b=\operatorname{EmptyBag}($ the carrier of $R$ ) if and only if the ordered partition of $b=\emptyset$. The theorem is a consequence of (32).

Let us consider $R$. The functor $\prec_{\mathcal{M}} R$ yielding a strict relational extension of (the carrier of $R)^{\otimes}$ is defined by

(Def. 13) for every elements $m, n$ of $i t, m \leqslant n$ iff $m \neq n$ and for every $x$ such that $m(x)>0$ holds $m(x)<n(x)$ or there exists $y$ such that $n(y)>0$ and $x \leqslant y$.

Let us note that $\prec_{\mathcal{M}} R$ is asymmetric and transitive.

Let us consider $I$. Let $R$ be a relation between $I$ and $I$. 
The functor LexOrder $(I, R)$ yielding a binary relation on $I^{*}$ is defined by

(Def. 14) for every $I$-valued finite sequences $p, q,\langle p, q\rangle \in$ it iff $p \subset q$ or there exists a natural number $k$ such that $k \in \operatorname{dom} p$ and $k \in \operatorname{dom} q$ and $\langle p(k)$, $q(k)\rangle \in R$ and for every natural number $n$ such that $1 \leqslant n<k$ holds $p(n)=q(n)$.

Let $R$ be a transitive binary relation on $I$. One can verify that $\operatorname{Lex} \operatorname{Order}(I, R)$ is transitive.

Let $R$ be an asymmetric binary relation on $I$. Note that $\operatorname{Lex} \operatorname{Order}(I, R)$ is asymmetric.

Now we state the proposition:

(60) Let us consider an asymmetric binary relation $R$ on $I$, and $I$-valued finite sequences $p, q, r$. Then $\langle p, q\rangle \in \operatorname{LexOrder}(I, R)$ if and only if $\langle r \frown p$, $\left.r^{\frown} q\right\rangle \in \operatorname{LexOrder}(I, R)$. The theorem is a consequence of (10).

Let us consider $R$. The functor $\prec \prec \mathcal{M} R$ yielding a strict relational extension of (the carrier of $R)^{\otimes}$ is defined by

(Def. 15) for every elements $m, n$ of $i t, m \leqslant n$ iff 〈the ordered partition of $m$, the ordered partition of $n\rangle \in \operatorname{LexOrder}\left(\left(\right.\right.$ the carrier of $\prec_{\mathcal{M}} R$ ), (the internal relation of $\left.\prec_{\mathcal{M}} R\right)$ ).

Observe that $\prec \prec \mathcal{M} R$ is asymmetric and transitive.

Now we state the propositions:

(61) Let us consider elements $a, b$ of the Dershowitz-Manna order $R$. Suppose $a \leqslant b$. Then $b \neq \operatorname{EmptyBag}($ the carrier of $R$ ). The theorem is a consequence of (29).

(62) Let us consider elements $a, b, c, d$ of the Dershowitz-Manna order $R$, and a bag $e$ of the carrier of $R$. Suppose $a \leqslant b$ and $e \mid a$ and $e \mid b$. If $c=a-^{\prime} e$ and $d=b-{ }^{\prime} e$, then $c \leqslant d$.

(63) Let us consider a (Bags $I$ )-valued finite sequence $p$, and an object $x$. Suppose $x \in I$ and $\left(\sum p\right)(x)>0$. Then there exists a natural number $i$ such that

(i) $i \in \operatorname{dom} p$, and

(ii) $p_{i}(x)>0$.

Proof: Define $\mathcal{P}$ [object] $\equiv$ for every (Bags $I$ )-valued finite sequence $p$ such that $p=\$_{1}$ and $\left(\sum p\right)(x)>0$ there exists a natural number $i$ such that $i \in \operatorname{dom} p$ and $p_{i}(x)>0 . \mathcal{P}[\emptyset]$ by $(32),[14,(7)]$. For every finite sequence $p$ and for every object $a$ such that $\mathcal{P}[p]$ holds $\mathcal{P}\left[p^{\frown}\langle a\rangle\right]$ by $(7)$, [4, (40)], [15, (25)], [6, (102)]. For every finite sequence $p, \mathcal{P}[p]$ from [4, Sch. 3].

(64) If $q$ is ordered and $q_{1}(x)=0$ and $b(x)>0$, then there exists $y$ such that $q_{1}(y)>0$ and $x \leqslant y$. 
Proof: Define $\mathcal{P}$ [natural number] $\equiv$ if $\$_{1} \in \operatorname{dom} q$, then for every $x$ such that $q_{\$_{1}}(x)>0$ there exists $y$ such that $q_{1}(y)>0$ and $x \leqslant y . \mathcal{P}[2]$ by $[15$, (25)]. For every natural number $i$ such that $2 \leqslant i$ and $\mathcal{P}[i]$ holds $\mathcal{P}[i+1]$ by [1, (11)], [15, (25)], [16, (3)]. For every natural number $i$ such that $i \geqslant 2$ holds $\mathcal{P}[i]$ from [1, Sch. 8]. Consider $i$ being a natural number such that $i \in \operatorname{dom} q$ and $q_{i}(x)>0$.

\section{REFERENCES}

[1] Grzegorz Bancerek. The fundamental properties of natural numbers Formalized Mathematics, 1(1):41-46, 1990.

[2] Grzegorz Bancerek. Reduction relations. Formalized Mathematics, 5(4):469-478, 1996.

[3] Grzegorz Bancerek. König's lemma Formalized Mathematics, 2(3):397-402, 1991.

[4] Grzegorz Bancerek and Krzysztof Hryniewiecki. Segments of natural numbers and finite sequences. Formalized Mathematics, 1(1):107-114, 1990.

[5] Czesław Byliński. Finite sequences and tuples of elements of a non-empty sets. Formalized Mathematics, 1(3):529-536, 1990.

[6] Czesław Byliński. Functions and their basic properties Formalized Mathematics, 1(1): 55-65, 1990.

[7] Czesław Byliński. Some basic properties of sets Formalized Mathematics, 1(1):47-53, 1990.

[8] Nachum Dershowitz. Orderings for term-rewriting systems. Theoretical Computer Science, 17(3):279-301, 1982. doi 10.1016/0304-3975(82)90026-3

[9] Nachum Dershowitz and Zohar Manna. Proving termination with multiset orderings. Communications of the ACM, 22(8):465-476, 1979. doi:10.1145/359138.359142.

[10] Gerard Huet and Derek C. Oppen. Equations and rewrite rules: A survey, Technical report, Stanford, CA, USA, 1980.

[11] Jean-Pierre Jouannaud and Pierre Lescanne. On multiset ordering. Information Processing Letters, 15(2):57-63, 1982. doi 10.1016/0020-0190(82)90107-7

[12] Robert Milewski. Natural numbers. Formalized Mathematics, 7(1):19-22, 1998.

[13] Eliza Niewiadomska and Adam Grabowski. Introduction to formal preference spaces. Formalized Mathematics, 21(3):223-233, 2013. doi 10.2478/forma-2013-0024.

[14] Andrzej Trybulec. Binary operations applied to functions Formalized Mathematics, 1 (2):329-334, 1990.

[15] Wojciech A. Trybulec. Non-contiguous substrings and one-to-one finite sequences Formalized Mathematics, 1(3):569-573, 1990.

[16] Wojciech A. Trybulec and Grzegorz Bancerek. Kuratowski - Zorn lemma Formalized Mathematics, 1(2):387-393, 1990.

Received December 31, 2015 